\title{
The Redundant Discrete Wavelet Transform and Additive Noise
}

\author{
James E. Fowler, Senior Member, IEEE
}

\begin{abstract}
The behavior under additive noise of the redundant discrete wavelet transform (RDWT), a frame expansion that is essentially an undecimated discrete wavelet transform, is studied. Known prior results in the form of inequalities bound distortion energy in the original signal domain from additive noise in frame-expansion coefficients. In this paper, a precise relationship between RDWT-domain and original-signal-domain distortion for additive white noise in the RDWT domain is derived.
\end{abstract}

Index Terms - redundant wavelet transform, frame expansion, additive noise

\section{INTRODUCTION}

It is often necessary to calculate distortion energy in the original signal domain from an equivalent quantity in the domain of a linear transform. That way, signal-processing operations can be performed in the transform domain with known effects in the original signal domain. For this reason, orthonormal sets are widely used, since, when the transform takes the form of an expansion using an orthonormal basis, Parseval's theorem guarantees $\|x\|^{2}=\|X\|^{2}$, or that the energy of the original signal $x$ in a Hilbert space can be determined from that of its transform $X$.

However, the constraints of orthonormal expansion sets can sometimes be too restrictive for some signal-processing applications. When one widens consideration to more general expansions, the increased functionality and flexibility often come at the cost of an exact energy relationship as above. Instead, one often has merely a bounding relationship in the form of

$$
A\|x\|^{2} \leq\|X\|^{2} \leq B\|x\|^{2}
$$

that frames the energy in one domain with respect to that of the other domain for some constants $A>0$ and $B<$ $\infty$. Expansions with such energy bounds are hence known as frame expansions.

One of the key benefits of the generality of a frame expansion lies in the robustness of the dual-frame, or pseudoinverse, frame reconstruction to added noise. Goyal et al. [1] show that, given a frame $\Psi=\left\{\psi_{n}\right\} \subset \mathbb{C}^{N}$ with $\left\|\psi_{n}\right\|^{2}=1$ and its dual $\widetilde{\Psi}=\left\{\widetilde{\psi}_{n}\right\}$, zero-mean white noise $X \in \mathbb{C}^{M}$ satisfies

$$
\frac{1}{B^{2}} E\left[\|X\|^{2}\right] \leq E\left[\|x\|^{2}\right] \leq \frac{1}{A^{2}} E\left[\|X\|^{2}\right],
$$

The author is with the Department of Electrical \& Computer Engineering, Mississippi State, Starkville, MS, and the Visualization, Analysis, and Imaging Laboratory (VAIL) within the GeoResources Institute (GRI) at the Mississippi State ERC.

This work was funded in part by the National Science Foundation under Grant No. CCR-0310864. where $x=\sum_{n} X[n] \widetilde{\psi}_{n}$ is the dual-frame reconstruction of $X$. If the frame is redundant (i.e., $M>N$ ), then $A>1$ [2]. In this case, less noise energy will result in the original signal domain from added noise in the domain of the redundant frame expansion.

In this paper, we focus on a specific redundant frame expansion known as the redundant discrete wavelet transform (RDWT), which is essentially an undecimated version of the discrete wavelet transform (DWT) ubiquitous to modern signal-processing applications. Since it is a frame expansion, the RDWT has energy bounds as in (1). As the initial contribution of this paper, we determine values for the frame-bound constants $A$ and $B$ assuming that an orthonormal filter pair underlies the RDWT. Then, as the primary contribution of this paper, we analyze the performance of the RDWT under additive noise. We find that, even though the RDWT is a highly redundant frame expansion, we can determine exactly the variance (i.e., expected distortion energy per sample) in the original signal domain of white noise added in the RDWT domain. Despite extensive use of the RDWT in prior signalprocessing applications, the frame-bound and noise-analysis results we present here are apparently new as we are unaware of their appearance in any prior literature.

The remainder of this paper is organized as follows. First, in Sec. II, we briefly overview the RDWT. The main contributions of the paper follow in Sec. III wherein we derive frame bounds for the RDWT, and in Sec. IV wherein we investigate the noise performance of the RDWT. Finally, we make some concluding remarks in Sec. V.

\section{The Redundant Discrete Wavelet Transform}

The RDWT ${ }^{1}$ has a long history, having been independently discovered a number of times and given a number of different names, including the algorithme à trous [4,5], the undecimated DWT (UDWT) [6], the overcomplete DWT (ODWT) [7], the shift-invariant DWT (SIDWT) [8], and discrete wavelet frames (DWF) [9]. There are several ways to implement the RDWT, and several ways to represent the resulting overcomplete set of coefficients. The original implementation was in form of the algorithme à trous [4,5], which, in essence, removes the downsampling operator from the usual implementation of the DWT. In this implementation, instead of signal downsampling, the filter responses themselves are upsampled, thereby inserting "holes" (trous in French) between nonzero filter taps.

Let $h \in \ell^{2}(\mathbb{Z})$ and $g \in \ell^{2}(\mathbb{Z})$ be the scaling and wavelet filters, respectively, of an orthonormal DWT. The RDWT

\footnotetext{
${ }^{1}$ Our use of the "RDWT" moniker is from [3].
} 
scaling filter at scale $j+1$ is defined recursively as

$$
h_{j+1}[k]=h_{j}[k] \uparrow 2= \begin{cases}h_{j}[k / 2], & k \text { even, } \\ 0, & k \text { odd, }\end{cases}
$$

where $h_{0}[k]=h[k]$. The wavelet filters $g_{j}[k]$ are defined similarly. The RDWT of $x \in \ell^{2}(\mathbb{Z})$ is then implemented recursively with the filter-bank operations

$$
\begin{aligned}
c_{j+1}[k] & =h_{j}[-k] * c_{j}[k], \\
d_{j+1}[k] & =g_{j}[-k] * c_{j}[k],
\end{aligned}
$$

where $c_{0}=x$ and $j=0, \ldots, J-1$. The $J$-scale RDWT is $X^{(J)}=\operatorname{RDWT}_{J}[x]=\left[\begin{array}{llll}c_{J} & d_{J} & \cdots & d_{1}\end{array}\right]$ such that

$$
\left\|X^{(J)}\right\|^{2}=\left\|c_{J}\right\|^{2}+\sum_{j=1}^{J}\left\|d_{j}\right\|^{2} .
$$

In the frequency domain, we have $\widehat{h}_{j}(\omega)=\widehat{h}_{0}\left(2^{j} \omega\right), \widehat{g}_{j}(\omega)=$ $\widehat{g}_{0}\left(2^{j} \omega\right)$,

$$
\begin{aligned}
& \widehat{c}_{j}(\omega)=\left[\prod_{\lambda=0}^{j-1} \widehat{h}_{0}^{*}\left(2^{\lambda} \omega\right)\right] \widehat{x}(\omega), \\
& \widehat{d}_{j}(\omega)=\widehat{g}_{0}^{*}\left(2^{j-1} \omega\right)\left[\prod_{\lambda=0}^{j-2} \widehat{h}_{0}^{*}\left(2^{\lambda} \omega\right)\right] \widehat{x}(\omega),
\end{aligned}
$$

and, since the filters $h$ and $g$ are orthonormal,

$$
\begin{array}{r}
\frac{1}{2 \pi} \int_{-\pi}^{\pi}\left|\prod_{\lambda=0}^{j-1} \widehat{h}_{0}\left(2^{\lambda} \omega\right)\right|^{2} d \omega=1, \\
\frac{1}{2 \pi} \int_{-\pi}^{\pi}\left|\widehat{g}_{0}\left(2^{j-1} \omega\right) \prod_{\lambda=0}^{j-2} \widehat{h}_{0}\left(2^{\lambda} \omega\right)\right| d \omega=1
\end{array}
$$

(see Sec. 7.3.3 of [2]). In order to reconstruct $x$ in the original signal domain given $X^{(J)}$ in the RDWT domain, one recursively performs the synthesis operation,

$$
c_{j}[k]=\frac{1}{2}\left(h_{j}[k] * c_{j+1}[k]+g_{j}[k] * d_{j+1}[k]\right) .
$$

The RDWT is a frame expansion, a fact we will verify below by calculating its frame bounds. The à trous synthesis procedure of (11) is the dual-frame reconstruction for this frame.

We note that an alternative implementation of the RDWT was independently proposed by Shensa [10] and Beylkin [11]. In essence, this implementation employs filtering and downsampling as in the usual critically sampled DWT; however, all "phases" of downsampled coefficients are retained and arranged as "children" of the signal that was decomposed. The process is repeated on all the lowpass bands to achieve multiple decomposition scales that form a "tree" of decompositions. Although this alternative tree-based RDWT is a useful and common implementation in practice, we will focus on the à trous implementation here since it is much more amenable to mathematical analysis and derivation, a characteristic we exploit as we study the noise properties of the RDWT in the next section.

\section{FRAME BOUndS OF THE RDWT}

Lemma 1 A single-scale RDWT operating in $\ell^{2}(\mathbb{Z})$ is a tightframe expansion with frame bounds $A=B=2$.

Proof: See Example 5.2 of [12].

Lemma 2 If $X^{(J)}$ is the J-scale RDWT of $x \in \ell^{2}(\mathbb{Z})$, then

$$
\left\|X^{(J)}\right\|^{2}=\left\|c_{J-1}\right\|^{2}+\left\|X^{(J-1)}\right\|^{2} .
$$

Proof: See App. I.

Theorem 1 A $J$-scale $R D W T$ operating in $\ell^{2}(\mathbb{Z})$ is a frame expansion with frame bounds $A=2$ and $B=2^{J}$.

Proof: By the definition of a frame, it is sufficient to show that the frame bounds exist to show that the RDWT is a frame. To establish frame bounds, we use a proof by induction. The inductive basis is given by Lemma 1 . The inductive step is as follows. Suppose that for $J \geq 2$, we have

$$
2\|x\|^{2} \leq\left\|X^{(J-1)}\right\|^{2} \leq 2^{J-1}\|x\|^{2}
$$

for $X^{(J-1)}=\left[\begin{array}{lllll}c_{J-1} & d_{J-1} & d_{J-2} & \cdots & d_{1}\end{array}\right]$, the $(J-1)$ scale RDWT of $x$. Then, for the $J$-scale RDWT, we have from Lemma 2 and (13), $\left\|X^{(J)}\right\|^{2}=\left\|c_{J-1}\right\|^{2}+\left\|X^{(J-1)}\right\|^{2} \geq$ $\left\|X^{(J-1)}\right\|^{2} \geq 2\|x\|^{2}$, which establishes inductively that the lower bound satisfies $A \geq 2$.

For the upper bound, we note that, from (6) and (13), we have $\left\|c_{J-1}\right\|^{2}=\left\|X^{(J-1)}\right\|^{2}-\sum_{j=1}^{J-1}\left\|d_{j}\right\|^{2} \leq 2^{J-1}\|x\|^{2}$. From Lemma 2 and (13) we then have $\left\|X^{(J)}\right\|^{2}=\left\|c_{J-1}\right\|^{2}+$ $\left\|X^{(J-1)}\right\|^{2} \leq 2^{J-1}\|x\|^{2}+2^{J-1}\|x\|^{2}=2^{J}\|x\|^{2}$, which establishes that the upper bound satisfies

$$
B \leq 2^{J} \text {. }
$$

In App. II, we show that the bounds of $A=2$ and $B=2^{J}$ are the tightest possible frame bounds since we can find sequences $x \in \ell^{2}(\mathbb{Z})$ that asymptotically meet these bounds.

\section{AdDitive NoIse IN THE RDWT Domain}

In this section, we consider zero-mean, white-noise signals $X$ in the RDWT domain, such that $E[X[n]]=0$ and $E\left[X[n] X^{*}[m]\right]=\sigma^{2}$ for $n=m$ and 0 otherwise. With the following theorems, we establish the effect of RDWT synthesis on this noise; we note that a similar procedure was used in [13] to analyze the critically sampled DWT. Throughout, $\mathbb{C}^{\infty}$ denotes the space of infinite-dimensional sequences.

Theorem 2 Suppose we have $X^{(J)}$ taking value in $\mathbb{C}^{\infty}$ such that $X^{(J)}=\left[\begin{array}{llll}c_{J} & d_{J} & \cdots & d_{1}\end{array}\right]$. Suppose a single subband of $X^{(J)}$ consists of zero-mean white noise of variance $\sigma^{2}$ while all the other subbands are zero. Then, the reconstruction $x$ due to the à trous synthesis algorithm of (11) is zero-mean noise with variance

$$
E\left[|x[k]|^{2}\right]=\frac{\sigma^{2}}{4^{j}}
$$

where $j$ is the scale of the subband in which the noise resides.

Proof: Establishing that $x$ has zero mean is straightforward, so we will focus on the variance. The noise in $X$ will be in 
either $c_{j}$ or $d_{j}$ while all the other subbands are zero. Let us consider first the case that noise is in $c_{j}$. Consequently, we have from (11),

$$
x[k]=c_{0}[k]=\left(\frac{1}{2}\right)^{j} h_{0}[k] * h_{1}[k] * \cdots * h_{j-1}[k] * c_{j}[k] .
$$

The power spectral density of the output of the synthesis operation will be

$$
S_{x}(\omega)=\frac{\sigma^{2}}{4^{j}}\left|\prod_{\lambda=0}^{j-1} \widehat{h}_{0}\left(2^{\lambda} \omega\right)\right|^{2},
$$

since the power spectral density of $c_{j}$ is $\sigma^{2}$, and $\widehat{h}_{j}(\omega)=$ $\widehat{h}_{0}\left(2^{j} \omega\right)$. Invoking (9), we have that the variance of $x$ is then

$$
E\left[|x[k]|^{2}\right]=\frac{1}{2 \pi} \int_{-\pi}^{\pi} S_{x}(\omega) d \omega=\frac{\sigma^{2}}{4^{j}} .
$$

For $d_{j}$, the proof is similar. In this case, we have from (11),

$$
x[k]=\left(\frac{1}{2}\right)^{j} h_{0}[k] * h_{1}[k] * \cdots * h_{j-2}[k] * g_{j-1}[k] * d_{j}[k],
$$

while the power spectral density is

$$
S_{x}(\omega)=\frac{\sigma^{2}}{4^{j}}\left|\widehat{g}_{0}\left(2^{j-1} \omega\right) \prod_{\lambda=0}^{j-2} \widehat{h}_{0}\left(2^{\lambda} \omega\right)\right|^{2} .
$$

Invoking (10), we have that the variance of $x$ is then

$$
E\left[|x[k]|^{2}\right]=\frac{1}{2 \pi} \int_{-\pi}^{\pi} S_{x}(\omega) d \omega=\frac{\sigma^{2}}{4^{j}} .
$$

Theorem 3 Suppose $X^{(J)}$ taking value in $\mathbb{C}^{\infty}$ is a zeromean, white-noise signal with variance $\sigma^{2}$. That is, suppose that the noise coefficients are mutually uncorrelated between subbands. Then, the reconstruction $x$ from (11) is zero-mean noise with variance

$$
E\left[|x[k]|^{2}\right]=\frac{\sigma^{2}}{3}\left[1+2\left(\frac{1}{4}\right)^{J}\right] .
$$

Proof: Because the noise in a given subband is uncorrelated from that in the other subbands, the output of the synthesis operation (11) for that subband will be uncorrelated from the synthesis outputs for the other subbands. Thus, the total variance of the output is the sum of the output variances due to each individual subband as given by Theorem 2. Consequently, we have

$$
E\left[|x[k]|^{2}\right]=\sigma^{2}\left(\frac{1}{4}\right)^{J}+\sigma^{2} \sum_{j=1}^{J}\left(\frac{1}{4}\right)^{j} .
$$

Using the fact that $\sum_{n=m}^{\infty} r^{n}=\frac{r^{m}}{1-r}$, for $|r|<1$, we have

$$
\begin{aligned}
E\left[|x[k]|^{2}\right] & =\sigma^{2}\left(\frac{1}{4}\right)^{J}+\frac{\sigma^{2}}{3}\left[1-\left(\frac{1}{4}\right)^{J}\right] \\
& =\frac{\sigma^{2}}{3}\left[1+2\left(\frac{1}{4}\right)^{J}\right] .
\end{aligned}
$$

\section{COnClusions}

Strictly speaking, the result of (2) applies only to finitedimensional spaces $\mathbb{C}^{N}$, whereas the frame bounds in Sec. III were derived assuming $\ell^{2}(\mathbb{Z})$, and the noise analysis of Sec. IV concerned white-noise signals in $\mathbb{C}^{\infty}$. If we ignore for the moment these space differences, (2) would suggest that the noise variance (expected energy per signal sample) in the original signal domain for white-noise $X^{(J)}$ with variance $\sigma^{2}$ is bounded as

$$
\frac{M \sigma^{2}}{B^{2} N} \leq \frac{1}{N} E\left[\|x\|^{2}\right] \leq \frac{M \sigma^{2}}{A^{2} N},
$$

assuming $x \in \mathbb{C}^{N}, X^{(J)} \in \mathbb{C}^{M}, E\left[\|X\|^{2}\right]=M \sigma^{2}$, and $x$ is reconstructed from $X^{(J)}$ with the à trous synthesis procedure of (11). For a $J$-scale RDWT, $M=(J+1) N$, and Theorem 1 indicates $A=2$ and $B=2^{J}$. Thus, we have

$$
\frac{(J+1) \sigma^{2}}{4^{J}} \leq \frac{1}{N} E\left[\|x\|^{2}\right] \leq \frac{(J+1) \sigma^{2}}{4} .
$$

We note that (26) suggests a limited ability to predict the effect in the original signal domain of noise added in the RDWT domain, particularly as $J$ becomes large. This observation conforms to our intuition concerning frames-since the frame bounds given by Theorem 1 widen as $J$ increases, we expect to be able to predict energy from one domain to the other with decreasing precision.

However, Theorem 3 tells us that we can make a much stronger characterization of the noise variance in the original signal domain than we are led to believe from (26). Theorem 3 indicates that, rather than being bounded by ever widening bounds, the noise variance actually is given by (22), approaching $\sigma^{2} / 3$ as $J$ becomes large.

We note that, although the preceding development focused on 1D signals, it is straightforward to generalize the derivations to the case of 2D image signals that are decomposed using a separable 2D RDWT. In this case, Theorem 3 generalizes to

$$
E\left[|x[k]|^{2}\right]=\frac{\sigma^{2}}{5}\left[1+4\left(\frac{1}{16}\right)^{J}\right] .
$$

In such image-processing applications, the RDWT is usually implemented with biorthogonal filters rather than orthonormal filters as assumed here. If the biorthogonal system is "nearorthonormal" as is often the case in practice (e.g., the ubiquitous 9-7 biorthogonal basis), then we will approximately have equality in (9) and (10), and consequently in (27).

Finally, in terms of application of the results presented here, we note that we have exploited (27) directly in an analysis concerning motion compensation in the RDWT domain for the RDWT-based coding of video in [14]. There, (27) is the keystone of a derivation that shows that multiple-phase motion compensation in the RDWT domain substantially outperforms an equivalent single-phase process, the inverse RDWT providing substantial reduction of the variance of the motion-compensation prediction residual as indicated by (27). Additionally, we suspect that the results here have analytical 
ramifications for RDWT-domain watermarking such as we previously considered in $[15,16]$; we are currently investigating this issue.

\section{APPENDIX I}

\section{PROOF OF LEMMA 2}

Consider the sum $\left\|c_{J}\right\|^{2}+\left\|d_{J}\right\|^{2}-\left\|c_{J-1}\right\|^{2}$. In the frequency domain, we have from (7) and (8),

$$
\begin{gathered}
\left\|\widehat{c}_{J}\right\|^{2}+\left\|\widehat{d}_{J}\right\|^{2}-\left\|\widehat{c}_{J-1}\right\|^{2}= \\
\frac{1}{2 \pi} \int_{-\pi}^{\pi}\left[\prod_{\lambda=0}^{J-1}\left|\widehat{h}_{0}\left(2^{\lambda} \omega\right)\right|^{2}+\left|\widehat{g}_{0}\left(2^{J-1} \omega\right)\right|^{2} \prod_{\lambda=0}^{J-2}\left|\widehat{h}_{0}\left(2^{\lambda} \omega\right)\right|^{2}-\right. \\
\left.\prod_{\lambda=0}^{J-2}\left|\widehat{h}_{0}\left(2^{\lambda} \omega\right)\right|^{2}\right]|\widehat{x}(\omega)|^{2} d \omega= \\
\frac{1}{2 \pi} \int_{-\pi}^{\pi}\left[\prod_{\lambda=0}^{J-2}\left|\widehat{h}_{0}\left(2^{\lambda} \omega\right)\right|^{2}\right]|\widehat{x}(\omega)|^{2} d \omega, \quad \text { (28) }
\end{gathered}
$$

where we use the fact that the filters are power complementary; i.e, $\left|\widehat{g}_{0}\left(2^{J-1} \omega\right)\right|^{2}+\left|\widehat{h}_{0}\left(2^{J-1} \omega\right)\right|^{2}=2$. Consequently, $\left\|\widehat{c}_{J}\right\|^{2}+\left\|\widehat{d}_{J}\right\|^{2}-\left\|\widehat{c}_{J-1}\right\|^{2}=\frac{1}{2 \pi} \int_{-\pi}^{\pi}\left|\widehat{c}_{J-1}(\omega)\right|^{2} d \omega=$ $\left\|\widehat{c}_{J-1}\right\|^{2}=\left\|c_{J-1}\right\|^{2}$, where we again employ (7). Thus, we have

$$
\left\|c_{J}\right\|^{2}+\left\|d_{J}\right\|^{2}-\left\|c_{J-1}\right\|^{2}=\left\|c_{J-1}\right\|^{2} .
$$

We then rearrange the sum in (6) as $\left\|X^{(J)}\right\|^{2}=\left\|c_{J}\right\|^{2}+$ $\sum_{j=1}^{J}\left\|d_{j}\right\|^{2}=\left\|c_{J}\right\|^{2}+\left\|d_{J}\right\|^{2}-\left\|c_{J-1}\right\|^{2}+\left\|X^{(J-1)}\right\|^{2}$, and we arrive at (12) by substituting (29) in for the first three terms.

\section{APPENDIX II \\ SignAls SATISFYING RDWT FRAME Bounds}

We now show that the bounds of $A=2$ and $B=2^{J}$ are the tightest possible frame bounds since we can find sequences $x \in \ell^{2}(\mathbb{Z})$ that asymptotically meet these bounds. Specifically, consider a constant sequence $x[k]=1$. Technically, this $x$ is not in $\ell^{2}(\mathbb{Z})$; however, we define $x_{N} \in \ell^{2}(\mathbb{Z})$ as $x_{N}[k]=\frac{1}{\sqrt{2 N+1}}$, for $-N \leq k \leq N$, and 0 otherwise. Clearly, $\left\|x_{N}\right\|^{2}=1, \forall N$. Since $h$ and $g$ are orthonormal filters, $h[-k] * x[k]=\sum_{n} h[n]=\sqrt{2}$, and $g[-k] * x[k]=$ $\sum_{n} g[n]=0$. Thus, we have in the limit,

$$
\begin{aligned}
& \lim _{N \rightarrow \infty} \sqrt{2 N+1}\left(h[-k] * x_{N}[k]\right)=h[-k] * x[k]=\sqrt{2} \\
& \lim _{N \rightarrow \infty} \sqrt{2 N+1}\left(g[-k] * x_{N}[k]\right)=g[-k] * x[k]=0 .
\end{aligned}
$$

Let $c_{j}[k]$ and $d_{j}[k]$ be the coefficient sequences produced by (4) and (5) with $c_{0}[k]=x_{N}[k]$. Then, from (30),

$$
\lim _{N \rightarrow \infty} c_{j}[k] \sqrt{2 N+1}=2^{j / 2}=2^{j / 2} \lim _{N \rightarrow \infty} x_{N}[k] \sqrt{2 N+1},
$$

and so

$$
\lim _{N \rightarrow \infty}\left\|c_{j}\right\|^{2}=2^{j} \lim _{N \rightarrow \infty}\left\|x_{N}\right\|^{2}=2^{j},
$$

since $\left\|x_{n}\right\|^{2}=1, \forall N$. Similarly, from (31), we have $\lim _{N \rightarrow \infty}\left\|d_{j}\right\|^{2}=0$.
Now, consider the quantity $\beta_{N}=\left\|X_{N}^{(J)}\right\|^{2} /\left\|x_{N}\right\|^{2}$, where $X_{N}^{(J)}=\operatorname{RDWT}_{J}\left[x_{N}\right]$. From (1), $\beta_{N} \leq B, \forall N$. From (6) and (33), we have

$$
\begin{aligned}
\lim _{N \rightarrow \infty} \beta_{N} & =\lim _{N \rightarrow \infty} \frac{\left\|X_{N}^{(J)}\right\|^{2}}{\left\|x_{N}\right\|^{2}}=\lim _{N \rightarrow \infty}\left\|X_{N}^{(J)}\right\|^{2} \\
& =\lim _{N \rightarrow \infty}\left[\left\|c_{J}\right\|^{2}+\sum_{j=1}^{J}\left\|d_{j}\right\|^{2}\right]=2^{J} .
\end{aligned}
$$

Consequently, we have $2^{J} \leq B$ and, from (14), $B \leq 2^{J}$. Thus, $B=2^{J}$

A similar derivation using $x[k]=(-1)^{k}$ and $x_{N}[k]=$ $\frac{(-1)^{k}}{\sqrt{2 N+1}}$ for $-N \leq k \leq N$ establishes that $A=2$.

\section{REFERENCES}

[1] V. K. Goyal, M. Vetterli, and N. T. Thao, "Quantized overcomplete expansions in $\mathbb{R}^{n}$ : Analysis, synthesis, and algorithms," IEEE Transactions on Information Theory, vol. 44, no. 1, pp. 16-31, January 1998.

[2] S. Mallat, A Wavelet Tour of Signal Processing. San Diego, CA: Academic Press, 1998.

[3] C. S. Burrus, R. A. Gopinath, and H. Guo, Introduction to Wavelets and Wavelet Transforms: A Primer. Upper Saddle River, NJ: Prentice-Hall, 1998.

[4] M. Holschneider, R. Kronland-Martinet, J. Morlet, and P. Tchamitchian, "A real-time algorithm for signal analysis with the help of the wavelet transform," in Wavelets: Time-Frequency Methods and Phase Space, J.M. Combes, A. Grossman, and P. Tchamitchian, Eds. Berlin, Germany: Springer-Verlag, 1989, pp. 286-297, proceedings of the International Conference, Marseille, France, December 14-18, 1987.

[5] P. Dutilleux, "An implementation of the "algorithme à trous" to compute the wavelet transform," in Wavelets: Time-Frequency Methods and Phase Space, J.-M. Combes, A. Grossman, and P. Tchamitchian, Eds. Berlin, Germany: Springer-Verlag, 1989, pp. 298-304, proceedings of the International Conference, Marseille, France, December 14-18, 1987.

[6] M. Lang, H. Guo, J. E. Odegard, C. S. Burrus, and R. O. Wells, Jr., "Noise reduction using an undecimated discrete wavelet transform," IEEE Signal Processing Letters, vol. 3, no. 1, pp. 10-12, January 1996.

[7] R. Zaciu, C. Lamba, C. Burlacu, and G. Nicula, "Image compression using an overcomplete discrete wavelet transform," IEEE Transactions on Consumer Electronics, vol. 42, no. 3, pp. 800-807, August 1996.

[8] M. Lang, H. Guo, J. E. Odegard, C. S. Burrus, and R. O. Wells, Jr., "Nonlinear processing of a shift invariant DWT for noise reduction," in Wavelet Applications II, H. H. Szu, Ed. Orlando, FL: Proc. SPIE 2491, April 1995, pp. 640-651.

[9] M. Unser, "Texture classification and segmentation using wavelet frames," IEEE Transactions on Image Processing, vol. 4, no. 11, pp. 1549-1560, November 1995.

[10] M. J. Shensa, "The discrete wavelet transform: Wedding the à trous and Mallat algorithms," IEEE Transactions on Signal Processing, vol. 40, no. 10, pp. 2464-2482, October 1992.

[11] G. Beylkin, "On the representation of operators in bases of compactly supported wavelets," SIAM Journal on Numerical Analysis, vol. 29, no. 6, pp. 1716-1740, December 1992.

[12] M. Vetterli and J. Kovačević, Wavelets and Subband Coding. Englewood Cliffs, NJ: Prentice-Hall, 1995.

[13] J. W. Woods and T. Naveen, "A filter based bit allocation scheme for subband compression of HDTV," IEEE Transactions on Image Processing, vol. 1, no. 3, pp. 436-440, July 1992.

[14] S. Cui, Y. Wang, and J. E. Fowler, "Motion compensation via redundantwavelet multihypothesis," IEEE Transactions on Image Processing, submitted March 2004, revised February 2005.

[15] J.-G. Cao, J. E. Fowler, and N. H. Younan, "An image-adaptive watermark based on a redundant wavelet transform," in Proceedings of the International Conference on Image Processing, vol. 2, Thessaloniki, Greece, October 2001, pp. 277-280.

[16] L. Hua and J. E. Fowler, "A performance analysis of spread-spectrum watermarking based on redundant transforms," in Proceedings of the IEEE International Conference on Multimedia and Expo, vol. 2, Lausanne, Switzerland, August 2002, pp. 553-556. 